\title{
A Pseudo-Bertrand Distribution for Time-Scale Analysis
}

\author{
Paulo Gonçalvés, Member, IEEE, and Richard G. Baraniuk, Member, IEEE
}

\begin{abstract}
Using the pseudo-Wigner time-frequency distribution as a guide, we derive two new time-scale representations: the pseudo-Bertrand and the smoothed pseudo-Bertrand distributions. Unlike the Bertrand distribution, these representations support efficient online operation at the same computational cost as the continuous wavelet transform. Moreover, they take advantage of the affine smoothing inherent in the sliding structure of their implementation to suppress cumbersome interference components.
\end{abstract}

\section{INTRODUCTION}

$\mathbf{T}$ THE time-scale distributions of the affine class [1]-[3] have proven to be a powerful alternative to the timefrequency distributions of Cohen's class [4] for the analysis of the time-varying spectral content of nonstationary signals. Just as time-frequency shift covariance is central to Cohen's class distributions, time-scale change covariance characterizes affine class distributions. That is, if $\Omega_{x}(t, f)$ is a time-scale distribution of the signal $x(t)$; then the distribution of the shifted and scaled signal $\frac{1}{\sqrt{\alpha}} x\left(\frac{t-t_{0}}{\alpha}\right)$ becomes $\Omega_{x}\left(\frac{t-t_{0}}{\alpha}, \alpha f\right)$. Affine covariance makes these new signal representations natural for a host of applications, including wideband radar and sonar and self-similar signal analysis.

The continuous wavelet transform ${ }^{1}$

$$
\begin{aligned}
D_{x}(t, f) & \equiv f^{\frac{1}{2}} \int x(\tau) \psi^{*}(f(\tau-t)) d \tau \\
& =f^{-\frac{1}{2}} \int X(\nu) \Psi^{*}\left(f^{-1} \nu\right) e^{i 2 \pi \nu t} d \nu
\end{aligned}
$$

and the scalogram [2], its squared magnitude, are certainly the most popular time-scale distributions. However, due to

Manuscript received July 18,1995 . This work was supported by the National Science Foundation under Grant MIP-9457438, by the Office of Naval Research under Grant N00014-95-1-0849, and by the State of Texas Advanced Technology Program under Grant TX-ATP 003604-002. The associate editor coordinating the review of this paper and approving it for publication was Prof. A. Tewfik.

P. Goncalvés is with the Department of Electrical and Computer Engineering, Rice University, Houston, TX 77251-1892 USA, on leave from Ecole Normale Superieure de Lyon, Lyon, France.

R. Baraniuk is with the Department of Electrical and Computer Engineering, Rice University, Houston, TX 77251-1892 USA

Publisher Item Identifier S 1070-9908(96)02740-X.

${ }^{1}$ Throughout this paper, we will employ the following notation: The variables $t$ and $f$ correspond to time and frequency, respectively; all integrals run from $-\infty$ to $+\infty$; lower-case letters denote time functions, and uppercase letters denote Fourier transforms. We will also consider only analytic signals, where $X(f) \equiv 0 \forall f<0$. Usually, the wavelet transform is expressed as a function of a time variable $t$ and a scale variable $a$. Here, we will use the reparametrization of scale as inverse frequency $a=f_{0} / f$ suggested in [2] and assume, without loss of generality, that the center frequency $f_{0}$ of the wavelet $\psi$ equals $1 \mathrm{~Hz}$. This interpretation of scale should not be confused with that of Cohen [4]. their linear structure, these tools are sensitive to the choice of the wavelet $\psi$ and lack certain desirable properties, such as simultaneously good time and frequency resolution and correct marginals.

To overcome these limitations, a broad class of bilinear distributions covariant to time and scale changes has been developed [1], [2]. Among these representations, the unitary Bertrand distribution [1]

$$
B_{x}(t, f) \equiv f \int \mu(u) X(\lambda(u) f) X^{*}(\lambda(-u) f) e^{i 2 \pi \xi(u) t f} d u
$$

with $\lambda(u)=\frac{-u}{e^{-u}-1}, \xi(u)=\lambda(u)-\lambda(-u)=u$, and $\mu(u)=$ $[\lambda(u) \lambda(-u)]^{\frac{1}{2}}\left(\frac{d \xi(u)}{d u}\right)^{\frac{1}{2}}$ plays a central role. In addition to having time-scale covariance, it is unitary, marginalizes to frequency when integrated over $t$ and to the Mellin transform when integrated over hyperbolic paths $t f=c$, and localizes on hyperbolic instantaneous frequencies and group delays.

Unfortunately, these desirable properties of the Bertrand distribution are offset by two major practical limitations. First, the entire signal enters into the calculation of the distribution at any point $(t, f)$ in the time-frequency plane, precluding its online operation with long signals. Second, due to its nonlinearity, interference components arise between each pair of signal components, complicating its interpretation [5], [6].

In this letter, we propose a solution to these problems: a pseudo-Bertrand distribution that not only offers asymptotically the same properties as the Bertrand distribution but also supports efficient online operation and suppresses troublesome cross components. Our derivation relies on the strong analogy between time-frequency and time-scale analysis and is inspired by the pseudo-Wigner distribution.

\section{ThE PSEUdo-Wigner Distribution}

While the short-time Foúrier transform

$$
S_{x}(t, f) \equiv \int x(\tau) w^{*}(\tau-t) e^{-i 2 \pi f \tau} d \tau
$$

and the spectrogram, its squared magnitude, are natural timefrequency representations, their dependence on the window function $w$ and subsequent lack of simultaneous time and frequency resolution have prompted the development of more advanced bilinear distributions, including the Wigner distribution [3], [4]

$$
W_{x}(t, f) \equiv \int x\left(t+\frac{\tau}{2}\right) x^{*}\left(t-\frac{\tau}{2}\right) e^{-i 2 \pi f \tau} d \tau .
$$


This representation overcomes most of the drawbacks associated with the spectrogram, but because it matches the window to the signal, it suffers from two major limitations of its own. First, it does not support online operation, since its calculation requires the entire signal. Second, its interpretation is complicated by nonlinear interference components.

Inserting a window function $h$ into (3) yields a sliding version of the Wigner distribution

$$
\widetilde{W}_{x}(t, f) \equiv \int x\left(t+\frac{\tau}{2}\right) x^{*}\left(t-\frac{\tau}{2}\right) h(\tau) e^{-i 2 \pi f \tau} d \tau
$$

known as the pseudo-Wigner distribution. Loosely speaking, this representation is equivalent to the Wigner distribution of the time windowed signal $x(\tau) \sqrt{h(2 \tau-t)}$, meaning that large amounts of data can be treated online. Alternatively, the pseudo-Wigner distribution can be written in terms of a convolution in frequency of two short-time Fourier transforms computed with window $w(\tau)=\sqrt{h(2 \tau)}[7]$

$$
\widetilde{W}_{x}(t, f)=2 \int S_{x}(t, f+\nu) S_{x}^{*}(t, f-\nu) d \nu .
$$

Since time windowing acts as a smoothing in the frequency domain, the pseudo-Wigner distribution suppresses the Wigner distribution interference components that oscillate in the frequency direction. Moreover, time direction smoothing can be implemented by limiting the range of the integral in (5) with a second lowpass function $G$

$$
\widetilde{W}_{x}(t, f)=2 \int G(\nu) S_{x}(t, f+\nu) S_{x}^{*}(t, f-\nu) d \nu .
$$

The result is called the smoothed pseudo-Wigner distribution. Choosing $G(\nu)=\delta(\nu)$ yields the spectrogram $\widetilde{W}_{x}(t, f)=$ $\left|S_{x}(t, f)\right|^{2}$.

\section{A PSEudo-Bertrand Distribution}

Using the results of the previous section as a guide, we now introduce a pseudo-Bertrand distribution. By rewriting (2) in the time domain

$$
\begin{aligned}
& B_{x}(t, f)=\int\left[(\lambda(u) f)^{\frac{1}{2}} \int x(\tau) e^{-i 2 \pi \lambda(u) f(\tau-t)} d \tau\right] \\
& \times\left[(\lambda(-u) f)^{\frac{1}{2}} \int x\left(\tau^{\prime}\right) e^{-i 2 \pi \lambda(-u) f\left(\tau^{\prime}-t\right)} d \tau^{\prime}\right]^{*} d u
\end{aligned}
$$

it is clear that the value of the Bertrand distribution at any point $(t, f)$ depends on the entire signal $x$. Since online operation requires that we consider the signal only in a sliding time interval, we introduce a window function $h$ in (7), and define

$$
\begin{aligned}
& \widetilde{B}_{x}(t, f) \equiv \int(\lambda(u) f)^{\frac{1}{2}}(\lambda(-u) f)^{\frac{1}{2}} \\
& {\left[\int x(\tau) h^{*}[\lambda(u) f(\tau-t)] e^{-i 2 \pi \lambda(u) f(\tau-t)} d \tau\right]} \\
& {\left[\int x\left(\tau^{\prime}\right) h^{*}\left[\lambda(-u) f\left(\tau^{\prime}-t\right)\right] e^{-i 2 \pi \lambda(-u) f\left(\tau^{\prime}-t\right)} d \tau^{\prime}\right]^{*} d u .}
\end{aligned}
$$

The dependence of $h$ on the analysis frequency $f$ guarantees $\widetilde{B}_{x}$ affine covariance to time shifts and scale changes. ${ }^{2}$ By analogy to the pseudo-Wigner distribution, we call this new time-scale representation the pseudo-Bertrand distribution.

The special structure of the pseudo-Bertrand distribution admits an efficient online implementation. Introducing the bandpass wavelet function $\psi(\tau)=h(\tau) e^{i 2 \pi \tau}$, we can reorder (8) to yield

$$
\widetilde{B}_{x}(t, f)=\int D_{x}(t, \lambda(u) f) D_{x}^{*}(t, \lambda(-u) f) d u
$$

where $D_{x}$ is the wavelet transform from (1) computed with wavelet $\psi$. This generalized convolution of two wavelet transforms parallels (5), which holds for the pseudo-Wigner distribution. An algorithm to compute the pseudo-Bertrand distribution runs as follows:

1) Compute the wavelet transform $D_{x}(t, f)$ with wavelet $\psi(\tau)=h(\tau) e^{i 2 \pi \tau}$. Samples should be spaced uniformly in time and exponentially in frequency.

2) At each time $t$, for a range of $u$, rescale $D_{x}(t, f)$ to $D_{x}(t, \lambda( \pm u) f)$ using the Mellin transform [8], which maps scale changes to simple phase shifts. Since the Mellin transform of a function $z(v)$ equals the Fourier transform of $e^{v / 2} z\left(e^{v}\right)$, a fast Fourier transform (FFT) applied to the exponentially spaced frequency samples of $D_{x}(t, f)$ implements a fast Mellin transform.

3) At each time $t$, compute the inner product (9) with respect to $u$.

Using a fast algorithm for the wavelet transform [8], [9], the computational cost of this procedure is $O(M N \log M)$ for $N$ time and $M$ frequency samples, ${ }^{3}$ which is on the same order as the cost for the spectrogram, pseudo-Wigner distribution, and scalogram.

In addition to being computationally efficient, the pseudoBertrand distribution suppresses interference components oscillating in the frequency direction, since the frequencydependent windowing in (8) acts as a constant- $Q$ frequency smoothing.

To suppress interference components oscillating in the time direction, we introduce a proportional-bandwidth time smoothing through a second lowpass function $G$

$$
\widetilde{B}_{x}(t, f)=\int G(u) D_{x}(t, f \lambda(u)) D_{x}^{*}(t, f \lambda(-u)) d u .
$$

We call this new time-scale representation the smoothed pseudo-Bertrand distribution. Choosing $G(u)=\delta(u)$ yields the scalogram $\widetilde{B}_{x}(t, f)=\left|D_{x}(t, f)\right|^{2}$.

In [10], we consider the existence of the pseudo-Bertrand distribution and the smoothed pseudo-Bertrand distribution within the affine class of time-scale representations. In particular, both distributions can be related to the important Bertrand

\footnotetext{
${ }^{2}$ Suppressing the $\lambda( \pm u)$ in $h$ in (8) yields an alternate pseudo-Bertrand distribution with identical covariance properties. However, this formulation does not appear to admit an efficient implementation. Rioul and Flandrin consider the same covariance requirements in their definition of the affine pseudo-Wigner distribution in [2].

${ }^{3}$ We assume that the length of the wavelet at maximum dilation is of $O(M)$.
} 


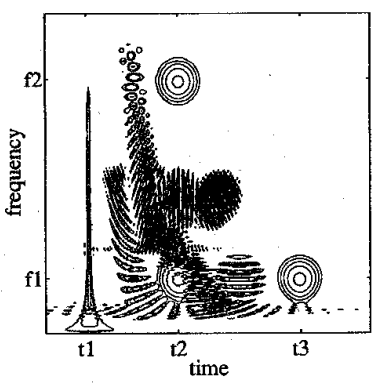

(a)

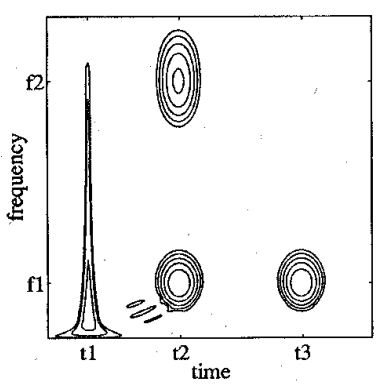

(c)

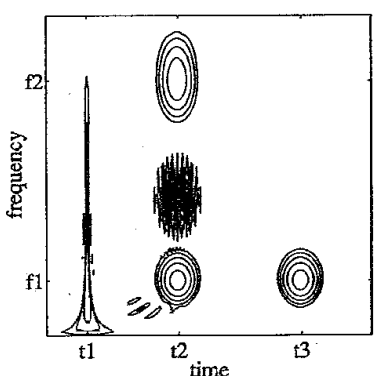

(b)

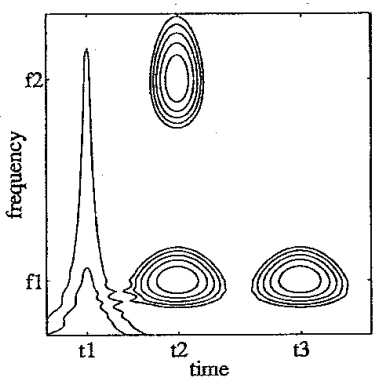

(d)
Fig. 1. Time-scale representations of a synthetic test signal composed of a Lipschitz singularity $\left|t-t_{1}\right|^{-0.15}$ followed by three Gaussian windowed tones. The frequency axis runs from 0.05 to 0.5 cycles/sample: (a) Bertrand distribution; (b) pseudo-Bertrand distribution computed with a Morlet wavelet of $Q=6$; (c) smoothed pseudo-Bertrand distribution computed with the same wavelet as in (b) and a square window $G$ of bandwidth 1.4 that time smooths with bandwidth $b(f)=1.4 f$; (d) scalogram computed with the same wavelet used in (b) and (c).

distribution by an affine smoothing of the form

$$
\widetilde{B}_{x}(t, f)=\iint B_{x}(\tau, \zeta) \Pi\left(f(\tau-t), \frac{\zeta}{f}\right) d \tau d \zeta
$$

with kernel function

$$
\begin{aligned}
\Pi(\tau, \zeta) & =\zeta \iint G(u) \frac{\mu(v)}{\mu(u)} \Psi\left(\zeta \frac{\lambda(v)}{\lambda(u)}\right) \Psi^{*}\left(\zeta \frac{\lambda(-v)}{\lambda(-v)}\right) \\
& \times e^{i 2 \pi \xi(v) \tau \zeta} d u d v .
\end{aligned}
$$

\section{EXAMPLE}

Fig. 1 illustrates several time-scale distributions of a synthetic test signal composed of a Lipschitz singularity followed by three modulated Gaussians. While the Bertrand distribution of Fig. 1(a) has excellent time-frequency resolution, it also has copious interference components. The constant- $Q$ frequency smoothing of the pseudo-Bertrand distribution of Fig. 1(b) suppresses the interference components that oscillate in the frequency direction without affecting the time resolution of the representation. The proportional-bandwidth time smoothing of the smoothed pseudo-Bertrand distribution of Fig. 1(c) suppresses the interference components that oscillate in the time direction. For comparison purposes, in Fig. 1(d), we plot the scalogram, which can also be obtained from the Bertrand distribution via an affine smoothing.

\section{CONCLUSIONS}

Although the Bertrand distribution has many attractive properties, lack of an efficient implementation has limited its impact on time-varying signal analysis. By overcoming some of its limitations, the pseudo-Bertrand and smoothed pseudoBertrand distributions should open up new application areas to this powerful tool. Moreover, since the Bertrand distribution belongs to a more general class of affine Wigner distributions of the form (2) with $\lambda_{k}(u)=\left[k\left(e^{-u}-1\right) /\left(e^{-k u}-1\right)\right]^{1 /(k-1)}$ [1], we can extend our methods and construct a class of pseudo-affine Wigner distributions. Members of this class, such as the pseudo-Unterberger and the pseudo-D distributions, gain efficient implementations [10]. Finally, to tune the pseudo-Bertrand distribution to the local characteristics of the signal, we can adapt the wavelet $\psi$ in the sliding algorithm using the techniques of [11].

\section{REFERENCES}

[1] J. Bertrand and P. Bertrand, "A class of affine Wigner functions with extended covariance properties," J. Math. Phys., vol. 33, no. 7, pp. 2515-2527, July 1992.

[2] O. Rioul and P. Flandrin, "Time-scale energy distributions: A general class extending wavelet transforms," IEEE Trans. Signal Processing, vol. 40 , no. 7 , pp. 1746-1757, July 1992.

[3] P. Flandrin, Temps-Fréquence. Paris: Hermés, 1993.

[4] L. Cohen, Time-Frequency Analysis. Englewood Cliffs, NJ: PrenticeHall, 1995.

[5] P. Flandrin and P. Gonçalvés, "Geometry of affine distributions," in Proc. IEEE Int. Symp. Time-Frequency Time-Scale Anal., Philadelphia; PA, 1994, pp. 80-83.

[6] P. Flandrin and P. Gonçalvés, "Geometry of affine time-frequency distributions," Appl. Comp. Harmonic Anal., to be published.

[7] L. Stankovic, "A method for time-frequency analysis," IEEE Trans. Sional Processing, vol. 42, no. 1, pp. 225-229, Jan. 1994.

[8] J. P. Ovarlez, J. Bertrand, and P. Bertrand, "Computation of affine timefrequency distributions using the fast Mellin transform," in Proc. IEEE Int. Conf. Acoust., Speech, Signal Processing, San Francisco, CA, 1992, pp. V117-V120.

[9] D. L. Jones and R. G. Baraniuk, "Efficient approximation of continuous wavelet transforms," Electron. Lett., vol. 27, no: 9, pp. 748-750, Apr. 1991.

[10] P. Gonçalvés and R. G. Baraniuk, "Pseudo affine Wigner distributions," in Proc. IEEE Int. Conf. Acoust. Speech, Signal Processing, Atlanta, GA, 1996.

[11] D. L. Jones and R. G. Baraniuk, "A simple scheme for adapting timefrequency representations," IEEE Trans. Signal Processing, vol. 42, no. 12, pp. 3530-3535, Dec. 1994. 